\title{
INDICADORES DE PRODUCTIVIDAD Y CALIDAD DE GEL DE SÁBILA EN CONDICIONES DE ESTRÉS SALINO
}

\section{PRODUCTIVITY AND QUALITY INDICATORS OF ALOE GEL UNDER SALINE STRESS CONDITIONS}

\author{
Nidia S. Sifuentes-Rodriguez ${ }^{1}$, Aurelio Pedroza-Sandoval ${ }^{1 *}$, \\ Jorge A. Zegbe ${ }^{2}$ y Ricardo Trejo-Calzada ${ }^{1}$
}

\begin{abstract}
'Universidad Autónoma Chapingo (UACh), Unidad Regional Universitaria de Zonas Áridas, Bermejillo, Durango, México. ${ }^{2}$ Instituto Nacional de Investigaciones Forestales, Agrícolas y Pecuarias, Campo Experimental Zacatecas, Calera de Víctor Rosales, Zacatecas, México.

*Autor de correspondencia (apedroza@chapingo.uruza.edu.mx)
\end{abstract}

\section{RESUMEN}

Las zonas áridas abarcan grandes regiones de México con problemas de escasez hídrica, suelos superficiales, pobres en materia orgánica y altos contenidos de sales. La sábila [Aloe vera (L.) Burm. f.] es una alternativa viable de producción ante condiciones ambientales limitantes. El objetivo de este estudio fue evaluar el efecto de la salinidad en el crecimiento de las hojas y calidad de gel de la sábila. El estudio consistió en probar seis tratamientos salinos: $0,500,1000,2000,4000$ y $8000 \mathrm{mg} \mathrm{L}^{-1}$ de $\mathrm{NaCl}$, correspondientes a conductividades eléctricas (CE) de 0.36 (agua de riego sin agregar $\mathrm{NaCl}$ ), $1.39,2.0,3.6,5.6$ y $7.6 \mathrm{dS} \mathrm{m}^{-1}$, respectivamente. El ensayo se condujo bajo un diseño experimental de bloques completos al azar con cuatro repeticiones. Las variables medidas fueron longitud de hoja, ancho de hoja, grosor de hoja, biomasa de la hoja, biomasa del gel, biomasa fresca y seca de la planta sin raíz, pH del gel, sólidos solubles totales y sólidos precipitables en metanol. La biomasa de la hoja y el contenido de gel fueron similares entre 0.36 y $3.6 \mathrm{dS}$ $\mathrm{m}^{-1}$, lo que sugirió cierto grado de tolerancia al estrés salino; sin embargo, a partir de los $5.6 \mathrm{dS} \mathrm{m}^{-1}$ hubo un efecto negativo en éstas y otras variables. La concentración de sólidos solubles totales y sólidos precipitables en metanol se incrementó en función de la $\mathrm{CE}$, dentro de cierto límite. Estos parámetros se consideran como un indicador de alto valor agregado desde el punto de vista comercial. Se confirma que ante condiciones moderadas de estrés, en este caso por salinidad, se favorece la calidad del gel, aunque se acompaña de un efecto negativo en el crecimiento y producción de hoja de sábila.

Palabras clave: Aloe barbadensis, calidad química del agua, salinidad agrícola, sólidos totales.

\section{SUMMARY}

Arid lands cover large regions of Mexico, with problems of water scarcity, shallow soils, poor organic matter content and high salt content. Aloe [Aloe vera (L.) Burm. f.] is a viable alternative for production under limiting environmental conditions. This study evaluated the effect of salinity on leaf growth and gel quality of aloe. The study consisted in testing six saline treatments: $0,500,1000,2000,4000$ and $8000 \mathrm{mg} \mathrm{L}^{-1}$ of $\mathrm{NaCl}$, corresponding to electrical conductivities $(\mathrm{EC})$ of 0.36 (irrigation water without adding $\mathrm{NaCl}$ ), $1.39,2.0,3.6,5.6$ and $7.6 \mathrm{dS} \mathrm{m}^{-1}$, respectively. The trial was conducted under a randomized complete blocks experimental design with four replications. The traits measured were leaf length, leaf width, leaf thickness, leaf biomass, gel biomass, fresh and dry biomass of the plant without root, gel pH, total soluble solids and solids precipitable in methanol. Leaf biomass and gel content were similar between 0.36 and $3.6 \mathrm{dS} \mathrm{m}^{-1}$, which suggested some degree of tolerance to salt stress; however, from $5.6 \mathrm{dS} \mathrm{m}^{-1}$, there was a negative effect on these and other traits. Total soluble solids concentration and precipitable solids in methanol increased as a function of EC, up to a certain limit. These parameters are considered an indicator of high added value from a commercial point of view. It is confirmed that under moderate stress conditions, in this case salinity, the quality of the gel is favored, but a negative effect accompanies growth and production of the aloe leaf.

Index words: Aloe barbadensis, agricultural salinity, chemical water quality, total solids.

\section{INTRODUCCIÓN}

La sábila [Aloe vera (L.) Burm. f.] (sinónimo Aloe barbadensis Mill.) es una especie nativa del mediterráneo, entre el sur de Europa y norte de África. Actualmente se encuentra distribuida en todo el mundo (Baruah et al., 2016). Los productos derivados de la sábila corresponden principalmente a extractos de polvo y gel, los cuales se comercializan en la industria cosmetológica, médica y alimenticia. Las especies $A$. vera y $A$. arborescens son las más extensamente cultivadas a nivel comercial (Lee, 2006; Ramachandra y Rao, 2008).

La hoja de sábila contiene más de 100 componentes bioactivos que, a través de una compleja interacción sinérgica entre ellos, confieren múltiples beneficios a la salud (Sánchez-Machado et al., 2017), por lo que ha sido una de las plantas más utilizadas en la historia de la humanidad (Lee, 2006). Los estudios económicos de productos derivados de la hoja de sábila indican que el nicho de mercado de mayor importancia actual y potencial se encuentra en países europeos y asiáticos (Pedroza y Gómez, 2006).

El tejido del mesófilo de las hojas suculentas de la sábila y su ruta fotosintética de metabolismo ácido crasuláceo (CAM, por sus siglas en inglés) (Borland et al., 2009) son características anatómico-fisiológicas que le permiten a la 
planta una gran capacidad de adaptación a las condiciones de estrés por sequía, al mantener potenciales hídricos altos en sus tejidos ante elevadas concentraciones de sales, altas temperaturas o déficit de humedad en el suelo (Grace et al., 2015; Pedroza y Gómez, 2006). De acuerdo con Jin et al. (2007), algunas especies de sábila pueden crecer normalmente ante condiciones de estrés salino sin afectar el crecimiento de manera significativa.

La salinidad edáfica se refiere a la cantidad de sales presentes en la solución del suelo y puede ser estimada indirectamente mediante la medición de la conductividad eléctrica (CE). El valor de CE es influenciado por la concentración y composición de las sales disueltas. A mayor valor de CE, mayor es la salinidad (GonzálezJiménez et al., 2020). Bajo condiciones de sequía o riegos deficitarios, la acumulación de sales en la superficie de los suelos aumenta, debido a que el agua aplicada en el riego, además de ser salina, no es capaz de lavar el exceso de sales, con el consecuente impacto negativo en la producción agrícola (FAO, 2009). El problema de la salinidad de los suelos aumenta por el inadecuado manejo de los agroecosistemas y hace que la agricultura se desplace hacia zonas marginales (Bazzigalupi et al., 2008). De esta manera, el estrés salino es considerado un problema de carácter global por el efecto negativo en el crecimiento y desarrollo de diferentes especies vegetales (Lata y Prasad, 2011; Olfati et al., 2012). Más del 20 \% de la superficie cultivable en el mundo se encuentra afectada por la salinidad (Gupta y Huang, 2014).

En las zonas áridas y semiáridas con áreas de riego, el agua es salina $y$, ante condiciones de alta evapotranspiración, se provoca la salinización de los suelos con un efecto colateral en la producción agrícola (Ritzema, 2016). La salinidad de los suelos en la Comarca Lagunera de los estados de Coahuila y Durango, México, que corresponde a la región de este estudio, es una condición abiótica generalizada por la deficiente calidad química del agua que se extrae de pozos profundos (Azpilcueta et al., 2017). El uso de cultivos alternativos tolerantes al estrés por salinidad de los suelos es una de las opciones a esta condición.

La sábila es un cultivo alternativo de alto potencial en regiones agrícolas donde la producción de los cultivos tradicionales es limitada por condiciones ambientales adversas, debido a que la planta posee una alta capacidad de adaptación a diferentes ambientes y tolerancia a diversos tipos de estrés, tanto de origen biótico como abiótico (Pedroza y Gómez, 2006). Además de considerarse como un cultivo alternativo en áreas marginales, los productos derivados de la sábila, como el jugo y el gel, son bien cotizados en el mercado nacional e internacional (Aba et al., 2016). La demanda de estos productos requiere de alto valor cualitativo; es decir, un alto contenido de polisacáridos, lo cual es posible cuando la planta se desarrolla en condiciones moderadas de algún tipo de estrés ambiental (Núñez-Colima et al., 2018); por lo anterior, este cultivo representa una alternativa productiva desde el punto de vista social, económico y ambiental.

Actualmente se continúa explorando el conocimiento y tecnología en diferentes cultivos con relación a la tolerancia a estrés ambiental, con la perspectiva de mitigar sus impactos y mantener la actividad productiva en condiciones adecuadas, de acuerdo con las necesidades agroalimentarias de cada región. El objetivo de este estudio fue determinar el efecto del estrés al aplicar agua salina al suelo en el crecimiento de la planta, la producción de biomasa y la calidad del gel de la hoja de sábila.

\section{MATERIALES Y MÉTODOS}

\section{Sitio del experimento y material vegetal}

El estudio se llevó a cabo de agosto a diciembre de 2016 en el Instituto Nacional de Investigaciones Forestales, Agrícolas y Pecuarias (INIFAP) en Matamoros, Coahuila, México, ubicado a $25^{\circ} 31^{\prime} 58^{\prime \prime} \mathrm{N}$ y $103^{\circ} 14^{\prime} 32^{\prime \prime}$ O, a 1150 msnm, con un promedio de precipitación anual de 240 $\mathrm{mm}$ y una temperatura que oscila entre 9 y $41^{\circ} \mathrm{C}$, con una media de $18^{\circ} \mathrm{C}$ (SMN, 2017). El experimento se realizó en condiciones de malla-sombra en estructura de $25 \times 40$ $\mathrm{m}$ abierta en los costados. La malla fue de color negro, con $90 \%$ de sombreado y $10 \%$ de transmisión de luz. Se usaron plantas de un promedio de $30 \mathrm{~cm}$ de altura al trasplante, con un periodo de aclimatación de dos meses (agosto y septiembre) y un mes de riego en los diferentes tratamientos (octubre).

\section{Tratamientos y diseño experimental}

Se probaron seis tratamientos salinos: 0 (testigo), 500, 1000, 2000, 4000 y $8000 \mathrm{mg} \mathrm{L}^{-1}$ de NaCl, lo cual correspondió a conductividades eléctricas (CE) de 0.36 (sin adición de

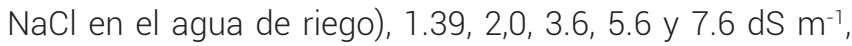
respectivamente. Estos tratamientos fueron aplicados al agua de riego al inicio del experimento. Se utilizó un diseño experimental de bloques completos al azar con cuatro repeticiones. Las unidades experimentales consistieron en macetas de $30 \mathrm{~L}$ de capacidad, con suelo de textura franco arenoso a un pH de 8.47, con plantas individuales. El riego fue de $2 \mathrm{~L}$ por semana por cada unidad experimental.

\section{Variables de respuesta}

Las variables se midieron durante los meses de 
noviembre y diciembre y éstas fueron: longitud de la hoja en $\mathrm{cm}$, mediante uso de cinta métrica; ancho y grosor de la hoja en cm, con uso de vernier (Truper, modelo 14388, China). Las variables relacionadas a la calidad de gel fueron: $\mathrm{pH}$, medido con un potenciómetro (Conductronic, modelo PH140, Puebla, México); concentración de azúcares en ${ }^{\circ}$ Brix, mediante un refractómetro (Atago ${ }^{\circledR}$, modelo PAL-1, Monterrey, N. L., México); concentración de sólidos totales y concentración de sólidos precipitables en metanol, determinados conforme a la metodología citada por Wang y Strong (1995), con base en los estándares de calidad internacional indicados por el International Aloe Science Council (IASC, 2008). La concentración de Mg, Na, $\mathrm{K}$ y Ca en $\mathrm{mg} \mathrm{L}^{-1}$ se cuantificó mediante espectrofotometría de absorción atómica por el método de flama, de acuerdo con la Norma Oficial Mexicana NOM-117-SSA1-1994 (SSA, 1995). Al final del experimento, mediante muestreo destructivo, se midió la biomasa de la hoja en g, la biomasa del gel en g, la biomasa fresca y seca de la planta sin raíz

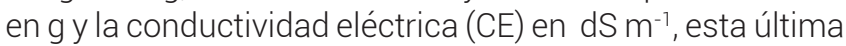
con el medidor de conductividad en extracto saturado del suelo (Horiba, modelo B-173, Querétaro, México).

\section{Análisis de datos}

Los datos se analizaron a través de un modelo lineal con el procedimiento GLM del programa Statistical Analysis System (SAS) versión 9.3, (SAS Institute, 2011). La comparación entre medias de tratamientos se realizó con la prueba del intervalo estudentizado de Tukey ( $\mathrm{P} \leq$ 0.05). Con el propósito de encontrar las asociaciones entre variables se realizó un análisis de correlación de Pearson, el cual se ejecutó mediante el procedimiento CORR con el mismo programa SAS.

\section{RESULTADOS Y DISCUSIÓN}

\section{Crecimiento de la hoja y producción de gel de sábila}

La longitud de la hoja de sábila fue estadísticamente similar entre tratamientos $(P \leq 0.05)$ (Cuadro 1). Lo anterior coincidió con lo indicado por Jin et al. (2007), quienes mencionaron que algunas variedades de sábila presentan tolerancia a la salinidad, e incluso que las plantas puedan ser irrigadas con el $50 \%$ de agua de mar sin afectar significativamente el crecimiento. Rahi et al. (2013) reportaron que plantas de sábila fueron capaces de sobrevivir, y aun incrementar la biomasa en fresco y en seco de las hojas, en condiciones de moderada sodicidad, de hasta $30 \%$ de sodio intercambiable.

En el presente estudio el ancho de la hoja fue afectado

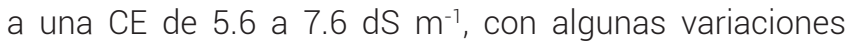
intermedias, mientras que el grosor de hoja fue más sensible a la salinidad, ya que disminuyó significativamente a partir de $3.6 \mathrm{dS} \mathrm{m}^{-1}$ (Cuadro 1). Tanto el ancho como el grosor de la hoja fueron afectados negativamente por el incremento de la salinidad. Lo anterior pudiera estar asociado con la dificultad para la absorción de agua y de nutrientes por la planta, lo que ocasionaría la disminución de la biomasa (Ashraf y Harris, 2004; Munns y Tester, 2008).

El umbral de tolerancia a la salinidad de la planta de sábila pudo ser más alto debido a que la concentración de sales fue mayor al final del experimento, por el contenido natural de $\mathrm{NaCl}$ en el agua de riego, de tal manera que al término del experimento la conductividad del suelo saturado fue de $2.32,5.17,7.82,10.9,20.47$ y $40.52 \mathrm{dS} \mathrm{m}^{-1}$ para los tratamientos, que inicialmente registraron una CE de $0.36,1.39,2.0,3.6,5.6,7.6 \mathrm{dS} \mathrm{m}^{-1}$, respectivamente (Cuadro 1), debido a la adición salina más el contenido natural de sal en el agua de riego. En el tratamiento de 3.6

Cuadro 1. Efecto de la salinidad en algunos atributos morfológicos de la hoja de sábila [Aloe vera (L.) Burm. f.].

\begin{tabular}{|c|c|c|c|c|}
\hline \multicolumn{2}{|c|}{ Conductividad eléctrica ( $\mathrm{dS} \mathrm{\textrm {m } ^ { - 1 } )}$} & \multicolumn{3}{|c|}{ Variables morfológicas (cm) } \\
\hline Tratamientos $^{\dagger}$ & Suelo $^{+t}$ & Longitud de la hoja & Ancho de la hoja & Grosor de la hoja \\
\hline 0.36 (Testigo) & 2.32 & $33.9 \pm 0.6 \mathrm{a}$ & $4.4 \pm 0.04 a$ & $2.0 \pm 0.03 \mathrm{a}$ \\
\hline 1.39 & 5.2 & $32.8 \pm 0.7 \mathrm{a}$ & $4.2 \pm 0.09 a b$ & $1.9 \pm 0.03 \mathrm{a}$ \\
\hline 2.0 & 7.82 & $32.2 \pm 0.5 \mathrm{a}$ & $4.0 \pm 0.1 \mathrm{bc}$ & $1.9 \pm 0.03 a b$ \\
\hline 3.6 & 10.9 & $33.9 \pm 0.5 a$ & $4.5 \pm 0.05 a$ & $1.8 \pm 0.02 \mathrm{bc}$ \\
\hline 5.6 & 20.5 & $32.0 \pm 0.3 \mathrm{a}$ & $3.8 \pm 0.07 c$ & $1.7 \pm 0.02 \mathrm{~cd}$ \\
\hline 7.6 & 40.5 & $33.0 \pm 0.6 \mathrm{a}$ & $4.0 \pm 0.09 b c$ & $1.7 \pm 0.04 d$ \\
\hline
\end{tabular}

Medias con la misma letra dentro de una misma columna son estadísticamente iguales (Tukey, $\mathrm{P} \leq 0.05) .{ }^{\dagger} \mathrm{y}^{\dagger+}$ son la conductividad eléctrica medida en el agua al aplicar el $\mathrm{NaCl}$ una sola vez al inicio del experimento y aquella medida en el suelo al final del experimento, respectivamente. 
dS $\mathrm{m}^{-1}$ la CE del suelo al final del experimento llegó a 10.9 dS $\mathrm{m}^{-1}$ y aún en esa condición la longitud y anchura de la hoja no fueron afectados negativamente, pero sí lo fue en mayor medida el grosor de la hoja. Esto indicó cierto grado de tolerancia de la sábila al estrés salino ya que, con base en la NOM-021-RECNAT-2000 (SEMARNAT, 2002), el suelo se considera moderadamente salino de 2.1 a $4.0 \mathrm{dS} \mathrm{m}^{-1}$, salino de 4.1 a $8.00 \mathrm{dS} \mathrm{m}^{-1}$, fuertemente salino de $8.1 \mathrm{a}$ $16.0 \mathrm{dS} \mathrm{m}^{-1} \mathrm{y}$ muy fuertemente salino $>16.0 \mathrm{dS} \mathrm{m}^{-1}$.

La biomasa de la hoja y del gel disminuyeron significativamente en las plantas tratadas con riego a una conductividad eléctrica de 5.6 y $7.6 \mathrm{dS} \mathrm{m}^{-1}$ (Cuadro 2). Estas mismas variables se mantuvieron sin diferencia estadística en tratamientos de CE hasta $3.6 \mathrm{dS} \mathrm{m}^{-1}$, lo cual sugirió que, en términos de producción de biomasa de la hoja y del gel, esta planta puede tolerar ese grado de salinidad.

No obstantelo anterior, a medida que seincrementó el nivel de salinidad, la biomasa de la planta en fresco disminuyó (Cuadro 2). Esta variable es uno de los componentes de crecimiento y producción más importantes en este cultivo, ya que puede afectar otras variables como la biomasa de la hoja y la cantidad de gel (Pedroza-Sandoval et al., 2015); ésto indica que la disminución de la disponibilidad de agua debido al contenido de $\mathrm{NaCl}$ en el suelo induce una menor absorción de nutrientes, lo cual pudiera disminuir la capacidad de crecimiento de la planta, producto de una reducción de la expansión celular por la pérdida de turgencia (Munns y Tester, 2008). La biomasa en fresco de la parte aérea de la planta de sábila fue significativamente mayor, en tanto que el tratamiento más alto de salinidad provocó la menor biomasa fresca con diferencias significativas, ambas respuestas con respecto al testigo $(P \leq 0.05)$; sin embargo, la biomasa fresca de las plantas regadas con soluciones de hasta $3.6 \mathrm{dS} \mathrm{m}^{-1}$ no tuvo una variación significativa (Cuadro 2).

La biomasa seca de las plantas disminuyó significativamente en el tratamiento con CE de $2.0 \mathrm{dS} \mathrm{m}^{-1}$, aunque los tratamientos con 3.6 y $5.6 \mathrm{dS} \mathrm{m}^{-1}$ tuvieron una biomasa seca estadísticamente igual al de las plantas testigo. El tratamiento con $7.6 \mathrm{dS} \mathrm{m}^{-1}$ produjo la menor biomasa seca y se diferenció significativamente del testigo (Cuadro 2). Adicional a lo anterior, es necesario considerar que la CE generada en el suelo por la concentración salina aplicada al agua fue de $40.52 \mathrm{dS} \mathrm{m}^{-1}$ en el tratamiento más alto de salinidad $\left(\mathrm{CE}=7.6 \mathrm{dS} \mathrm{m}^{-1}\right)$, correspondiente a un suelo extremadamente salino, de acuerdo con la NOM021-RECNAT-2000 (SEMARNAT, 2002). En el tratamiento donde se inició la aplicación del $\mathrm{NaCl}\left(\mathrm{CE}=1.39 \mathrm{dS} \mathrm{\textrm {m } ^ { - 1 }}\right.$ ), la CE en el suelo fue de $5.17 \mathrm{dS} \mathrm{m}^{-1}$, que corresponde a un suelo ligeramente salino; en este caso, la diferencia en la biomasa seca correspondió a una reducción de sólo 5.7 \% con respecto del testigo. Lo anterior confirmó que la sábila presenta amplias perspectivas en sistemas agrícolas marginales, donde la salinidad sea uno de los factores limitantes de la producción (Pedroza et al., 2009)

Los resultados anteriores no concuerdan con los reportados por otros autores (García et al., 2014; Moghbeli et al., 2012; Murillo-Amador et al., 2015; Olfati et al., 2012), quienes observaron baja tolerancia de la sábila a la salinidad, puesto que a partir de bajas concentraciones de sales, disminuyó significativamente el número de hojas por planta, la biomasa de las mismas, biomasa y ancho de la hoja y el contenido de gel, entre otras características. En cambio, en el presente estudio se observó cierta tolerancia de la sábila al ser irrigada con niveles moderados de salinidad en el agua. Esta característica de tolerancia pudiera estar asociada con la ruta fotosintética CAM, la cual le permite mantener potenciales hídricos altos en los tejidos ante algún estrés abiótico moderado, como

Cuadro 2. Efecto de la salinidad en diferentes variables de productividad de hoja y gel de sábila [Aloe vera (L.) Burm. f.].

\begin{tabular}{lcccc}
\hline CE $\left(\mathrm{dS} \mathrm{m}^{-1}\right)$ & $\begin{array}{c}\text { Biomasa fresca de la } \\
\text { planta }^{++}(\mathrm{g})\end{array}$ & $\begin{array}{c}\text { Biomasa seca } \\
\text { de la planta }{ }^{++}(\mathrm{g})\end{array}$ & Biomasa de la hoja $(\mathrm{g})^{\text {Biomasa del gel }(\mathrm{g})}$ \\
\hline $0.36^{+}$ & $1205.8 \pm 128.42 \mathrm{a}$ & $57.5 \pm 5.52 \mathrm{a}$ & $178.0 \pm 23.944 \mathrm{a}$ & $100.3 \pm 18.100 \mathrm{a}$ \\
1.39 & $1140.0 \pm 90.74 \mathrm{ab}$ & $54.2 \pm 3.32 \mathrm{ab}$ & $161.0 \pm 28.891 \mathrm{ab}$ & $92.1 \pm 18.303 \mathrm{ab}$ \\
2.0 & $1008.0 \pm 214.76 \mathrm{abc}$ & $41.7 \pm 11.37 \mathrm{bc}$ & $161.0 \pm 18.617 \mathrm{ab}$ & $84.5 \pm 14.953 \mathrm{ab}$ \\
3.6 & $957.8 \pm 122.17 \mathrm{abc}$ & $52.2 \pm 7.39 \mathrm{ab}$ & $150.1 \pm 9.702 \mathrm{ab}$ & $86.5 \pm 6.553 \mathrm{ab}$ \\
5.6 & $687.5 \pm 52.43 \mathrm{bc}$ & $42.5 \pm 1.84 \mathrm{abc}$ & $88.1 \pm 12.840 \mathrm{~b}$ & $42.03 \pm 8.134 \mathrm{~b}$ \\
7.6 & $633.3 \pm 199.43 \mathrm{c}$ & $28.7 \pm 10.98 \mathrm{c}$ & $76.7 \pm 12.479 \mathrm{~b}$ & $35.90 \pm 9.186 \mathrm{~b}$ \\
\hline
\end{tabular}

Medias con la misma letra dentro de una misma columna son estadísticamente iguales (Tukey, $\mathrm{P} \leq 0.05$ ). CE: conductividad eléctrica medida en el agua al aplicar el $\mathrm{NaCl}$ una sola vez al inicio del experimento. ${ }^{\dagger}$ Testigo (sin agregar $\mathrm{NaCl}$, sólo con el contenido natural en el agua de riego). ${ }^{++}$Correspondiente a la biomasa fresca y seca del total de hojas de la planta de sábila. 
el inducido por la salinidad (Borland et al., 2009; Nobel y Zhang, 1997; Winter et al., 2005).

\section{Características químicas del gel}

El pH no mostró cambios significativos $(P \leq 0.05)$ debido al efecto de tratamientos (Cuadro 3); no obstante, los valores registrados, que variaron entre 3.95 y 4.09, son similares a los consignados por Choi y Chung (2003) para geles empleados como materia prima en la elaboración de jugo de sábila. La concentración de azúcares y el porcentaje de sólidos solubles totales se incrementaron significativamente $(P \leq 0.05)$ cuando la CE fue de 7.6 dS $\mathrm{m}^{-1}$; un comportamiento similar ocurrió para el caso de los sólidos precipitables en metanol. Estas variables se integran en la denominación de contenido de polisacáridos, la cual determina la calidad de los derivados de la hoja de sábila; ésto sugiere que, con determinado nivel de estrés, se afecta la producción de hoja de sábila, pero se mejora la calidad del gel, acorde con los estándares establecidos por el IASC (2008).
Se ha reportado que, por efecto de la salinidad, el crecimiento de las plantas se reduce por efecto, dependiente $\mathrm{o}$ independiente, de la acumulación de iones en la planta (Roy et al., 2014). Es probable que el principal efecto de la salinidad en el crecimiento de la sábila, en este estudio, se haya debido a los efectos independientes por la acumulación de iones en los tejidos, ya que, aunque con cierto grado de tolerancia, el $\mathrm{K}$ se incrementó significativamente $(P \leq 0.05)$ a partir de una CE de 5.6 dS $\mathrm{m}^{-1}$ y el $\mathrm{Na}$ y el $\mathrm{Mg}$ fueron significativamente superiores en la CE más alta $\left(7.6 \mathrm{dS} \mathrm{m}^{-1}\right)$, en tanto que el Ca tuvo valores similares entre tratamientos (Cuadro 4). Estos resultados difirieron parcialmente de aquellos encontrados por García et al. (2014), quienes reportaron que la salinidad incrementó el contenido iónico de todos los elementos antes citados, excepto K, el cual disminuyó significativamente. En este estudio K, junto con $\mathrm{Na}$ y $\mathrm{Mg}$ se incrementaron en niveles moderadamente altos de salinidad, posiblemente por mecanismos pasivos como el proceso de ajuste osmótico dentro de la planta.

Cuadro 3. Efecto de la salinidad en diferentes variables relacionadas a la calidad del gel de hoja de sábila [Aloe vera (L.) Burm. f.].

\begin{tabular}{|c|c|c|c|c|}
\hline \multirow[b]{2}{*}{$\mathrm{CE}\left(\mathrm{dS} \mathrm{m} \mathrm{m}^{-1}\right)$} & \multicolumn{4}{|c|}{ Características químicas del gel } \\
\hline & $\mathrm{pH}$ & $\begin{array}{l}\text { Concentración de } \\
\text { azúcares ('Brix) }\end{array}$ & Sólidos totales (\%) & $\begin{array}{c}\text { Sólidos precipitables en } \\
\text { metanol }(\%)\end{array}$ \\
\hline $0.36^{+}$ & $4.09 \pm 0.045 a$ & $0.80 \pm 0.033 b$ & $0.754 \pm 0.0443 b$ & $0.322 \pm 0.0328 c$ \\
\hline 1.39 & $3.95 \pm 0.032 \mathrm{a}$ & $0.80 \pm 0.001 b$ & $0.788 \pm 0.04781 b$ & $0.352 \pm 0.07048 \mathrm{bc}$ \\
\hline 2.0 & $3.99 \pm 0.060 \mathrm{a}$ & $0.85 \pm 0.028 b$ & $0.790 \pm 0.0465 b$ & $0.451 \pm 0.0628 \mathrm{bc}$ \\
\hline 3.6 & $3.90 \pm 0.050 \mathrm{a}$ & $0.90 \pm 0.040 \mathrm{~b}$ & $1.021 \pm 0.0528 b$ & $0.512 \pm 0.0903 \mathrm{bc}$ \\
\hline 5.6 & $4.00 \pm 0.103 a$ & $0.97 \pm 0.047 b$ & $1.113 \pm 0.0501 a b$ & $0.756 \pm 0.1556 \mathrm{ab}$ \\
\hline 7.6 & $4.00 \pm 0.123 a$ & $1.25 \pm 0.064 \mathrm{a}$ & $1.499 \pm 0.2010 a$ & $1.015 \pm 0.1159 a$ \\
\hline
\end{tabular}

Medias con la misma letra dentro de una misma columna son estadísticamente iquales (Tukey, P $\leq 0.05$ ). CE: Conductividad eléctrica medida en el agua al aplicar el $\mathrm{NaCl}$ una sola vez al inicio del experimento. ${ }^{\dagger}$ Testigo ( $\sin$ agregar $\mathrm{NaCl}$, sólo con el contenido natural en el agua de riego).

Cuadro 4. Efecto de la salinidad en la concentración de $\mathrm{Mg}, \mathrm{Na}, \mathrm{K}$ y Ca en el gel de sábila [Aloe vera (L.) Burm. f.].

\begin{tabular}{lcccc}
\hline $\mathrm{CE}\left(\mathrm{dS} \mathrm{m} \mathrm{m}^{-1}\right)$ & $\mathrm{Mg}\left(\mathrm{mg} \mathrm{L}^{-1}\right)$ & $\mathrm{Na}\left(\mathrm{mg} \mathrm{L}^{-1}\right)$ & $\mathrm{K}\left(\mathrm{mg} \mathrm{L}^{-1}\right)$ & $\mathrm{Ca}\left(\mathrm{mg} \mathrm{L}^{-1}\right)$ \\
\hline $0.36^{+}$ & $0.9 \pm 0.1019 \mathrm{~b}$ & $11.77 \pm 0.8871 \mathrm{~b}$ & $2.99 \pm 0.3142 \mathrm{~b}$ & $1.26 \pm 0.1999 \mathrm{a}$ \\
1.39 & $1.1 \pm 0.1530 \mathrm{ab}$ & $11.48 \pm 1.9212 \mathrm{~b}$ & $2.28 \pm 0.2771 \mathrm{~b}$ & $1.37 \pm 0.2206 \mathrm{a}$ \\
2.0 & $1.3 \pm 0.0594 \mathrm{ab}$ & $11.93 \pm 2.4210 \mathrm{~b}$ & $2.65 \pm 0.0944 \mathrm{~b}$ & $1.39 \pm 0.0771 \mathrm{a}$ \\
3.6 & $1.2 \pm 0.0969 \mathrm{ab}$ & $9.63 \pm 2.1387 \mathrm{~b}$ & $2.90 \pm 0.1956 \mathrm{~b}$ & $1.60 \pm 0.2516 \mathrm{a}$ \\
5.6 & $1.2 \pm 0.1454 \mathrm{ab}$ & $17.64 \pm 3.9806 \mathrm{~b}$ & $6.12 \pm 0.9833 \mathrm{a}$ & $1.17 \pm 0.1536 \mathrm{a}$ \\
7.6 & $1.6 \pm 0.2283 \mathrm{a}$ & $33.06 \pm 1.6564 \mathrm{a}$ & $6.59 \pm 1.0213 \mathrm{a}$ & $2.14 \pm 0.4316 \mathrm{a}$ \\
\hline
\end{tabular}

Medias con la misma letra dentro de una misma columna son estadísticamente iguales (Tukey $(\mathrm{P} \leq 0.05)$. CE: conductividad eléctrica medida en el agua al aplicar el $\mathrm{NaCl}$ una sola vez al inicio del experimento. ${ }^{\top}$ Testigo (sin agregar $\mathrm{NaCl}$, sólo con el contenido natural en el agua de riego). 
Se identificó una correlación negativa $(P \leq 0.05)$ entre la concentración de los sólidos solubles totales y los sólidos precipitables en metanol del gel, con respecto al crecimiento y producción de biomasa de la hoja. Lo anterior confirma que por efecto del estrés por salinidad la producción disminuye en cantidad pero mejora la calidad de la misma. Las correlaciones positivas de la biomasa fresca y seca de la planta, en relación con la biomasa fresca y seca de la hoja, se explica por la dependencia entre estas variables (Cuadro 5).

\section{CONCLUSIONES}

Aloe vera (L.) Burm. f. mostró cierto grado de tolerancia a la salinidad, reflejado en la producción de biomasa, producción de gel y una mayor concentración de solidos solubles y sólidos precipitables en metanol, con un moderado impacto negativo en el crecimiento y desarrollo de la hoja. Se promovió una mayor concentración iónica de los elementos K, Na y Mg en función directa de la salinidad, dentro de ciertos límites. Lo anterior confiere grandes perspectivas a la sábila como cultivo alternativo en áreas agrícolas marginales por efecto de la salinidad y otros factores limitantes. Puesto que es un producto de estrés moderado por salinidad, se puede promover una mayor calidad de gel al favorecer la concentración de polisacáridos como un parámetro de alto valor comercial en el mercado.

\section{AGRADECIMIENTOS}

Se extiende un agradecimiento al Centro Regional de Investigación Norte Centro del Instituto Nacional de Investigaciones Forestales Agrícolas y Pecuarias por las facilidades prestadas para llevar a cabo esta investigación.

\section{BIBLIOGRAFÍA}

Aba G. C. G., A. Pedroza S., R. Trejo C., I. Sánchez C., J. A. Samaniego G. y J. A. Chávez R. (2016) Uso de biofertilizantes en la producción de sábila Aloe vera (L.) L. N. Burm y calidad de gel. Investigación y Ciencia 24:26-32.

Ashraf M. and P. J. C. Harris (2004) Potential biochemical indicators of salinity tolerance in plants. Plant Science 166:3-16, https://doi. org/10.1016/j.plantsci.2003.10.024

Azpilcueta P. M. E., A. Pedroza S., I. Sánchez C, M. R. Salcedo J. y R. Trejo C. (2017) Calidad química del agua en un área agrícola de maíz forrajero (Zea mays L.) en la Comarca Lagunera, México. Revista Internacional de Contaminación Ambiental 33:75-83, https://doi.org/10.20937/RICA.2017.33.01.07

Baruah A., M. Bordoloi and H. P. D. Baruah (2016) Aloe vera: a multipurpose industrial crop. Industrial Crops and Products 94:951-963, https://doi.org/10.1016/j.indcrop.2016.08.034

Bazzigalupi 0., S. M. Pistorale y A. N. Andrés (2008) Tolerancia a la salinidad durante la germinación de semillas provenientes de poblaciones naturalizadas de agropiro alargado (Thinopyrum ponticum). Ciencia e Investigación Agraria 35:277-285, https:// doi.org/10.4067/S0718-16202008000300005

Borland A. M., H. Griffiths, J. Hartwell and J. A. C. Smith (2009) Exploiting the potential of plants with crassulacean acid metabolism for bioenergy production on marginal lands. Journal of Experimental Botany 60:2879-2896, https://doi.org/10.1093/ jxb/erp118

Choi S. and M. H. Chung (2003) A review on the relationship between Aloe vera components and their biologic effects. Seminars in Integrative Medicine 1:53-62, https://doi.org/10.1016/S1543-

Cuadro 5. Matriz de coeficientes de correlación de Pearson entre las características de calidad del gel, la biomasa de hoja y de la parte aérea de la planta y la cantidad de gel en Aloe vera (L.) Burm. f. ( $n=24)$.

\begin{tabular}{|c|c|c|c|c|c|c|c|}
\hline Variable & ST (\%) & SPM (\%) & $\mathrm{pH}$ & CA ( ${ }^{\circ}$ Brix $)$ & $\mathrm{BH}(\mathrm{cm})$ & $B G(g)$ & BFP (\%) \\
\hline SPM & $\begin{array}{c}0.88 \\
0.0001^{+}\end{array}$ & & & & & & \\
\hline $\mathrm{pH}$ & $\begin{array}{c}0.36 \\
0.813\end{array}$ & $\begin{array}{c}0.36 \\
0.0833\end{array}$ & & & & & \\
\hline $\mathrm{CA}$ & $\begin{array}{c}0.89 \\
0.0001\end{array}$ & $\begin{array}{c}0.77 \\
0.0001\end{array}$ & $\begin{array}{c}0.28 \\
0.1766\end{array}$ & & & & \\
\hline $\mathrm{BH}$ & $\begin{array}{c}-0.72 \\
0.0001\end{array}$ & $\begin{array}{c}-0.61 \\
0.0014\end{array}$ & $\begin{array}{c}-0.31 \\
0.1384\end{array}$ & $\begin{array}{c}-0.71 \\
0.0001\end{array}$ & & & \\
\hline$B G$ & $\begin{array}{l}-0.68 \\
0.0002\end{array}$ & $\begin{array}{c}-0.58 \\
0.0027\end{array}$ & $\begin{array}{c}-0.37 \\
0.0743\end{array}$ & $\begin{array}{c}-0.65 \\
0.0005\end{array}$ & $\begin{array}{c}0.95 \\
0.0001\end{array}$ & & \\
\hline BFP & $\begin{array}{l}-0.70 \\
0.0001\end{array}$ & $\begin{array}{c}-0.67 \\
0.0003\end{array}$ & $\begin{array}{l}-0.40 \\
0.0497\end{array}$ & $\begin{array}{c}-0.52 \\
0.0086\end{array}$ & $\begin{array}{c}0.80 \\
0.0001\end{array}$ & $\begin{array}{c}0.85 \\
0.0001\end{array}$ & \\
\hline BSP & $\begin{array}{l}-0.48 \\
0.0176\end{array}$ & $\begin{array}{c}-0.48 \\
0.0167\end{array}$ & $\begin{array}{c}-0.42 \\
0.0388\end{array}$ & $\begin{array}{l}-0.22 \\
0.302\end{array}$ & $\begin{array}{c}0.57 \\
0.0032\end{array}$ & $\begin{array}{c}0.65 \\
0.0005\end{array}$ & $\begin{array}{c}0.91 \\
0.0001\end{array}$ \\
\hline
\end{tabular}

†La cifra debajo de cada coeficiente de correlación corresponde al valor p para indicar nivel de significancia. ST: sólidos totales, SPM: sólidos precipitables en metanol, pH: pH del gel, CA: contenido de azúcares, BO: biomasa de la hoja, BG: biomasa del gel, BFP. biomasa fresca de la planta, BSP. biomasa seca de planta. 
1150(03)00005-X

FAO, Food and Agricultural Organization (2009) El manejo de suelos afectados por salinidad. Food and Agriculture Organization of the United Nations. Rome. http://www.fao.org/soils-portal/ soil-management/manejo-de-suelos-problematicos/suelosafectados-por-salinidad/es/ (September 2016)

García R. M. E., V. A. Franco-Salazar y J. A. Véliz (2014) Crecimiento y contenido iónico de Aloe vera (L.) Burm. f. (Sábila) bajo diferentes concentraciones de NaCl. Saber 26:385-394, https:// doi.org/10.13140/RG.2.1.2416.2723

González-Jiménez S. L., A. M. Castillo-González, M. R. García-Mateos, L. A. Valdez-Aguilar, C. Ybarra-Moncada y E. Avitia-García (2020) Respuesta de fresa cv. Festival a la salinidad. Revista Fitotecnia Mexicana 43:53-60, https://doi.org/10.35196/rfm.2020.1.5360

Grace 0. M., S. Buerki, M. R. E. Symonds, F. Forest, A. E. van Wyk, G. F. Smith, ... and N. Rønsted (2015) Evolutionary history and leaf succulence as explanations for medicinal use in aloes and the global popularity of Aloe vera. BMC Evolutionary Biology 15:29, https:// doi.org/10.1186/s12862-015-0291-7

Gupta B. and B. Huang (2014) Mechanisms of salinity tolerance in plants: physiological, biochemical and molecular characterization International Journal of Genomics 2014:701596, https://doi. org/10.1155/2014/701596

IASC, International Aloe Science Council (2008) Aloe Scientific Primer. International Aloe Science Council. Silver Spring, Maryland, USA. 17 p. http://allaboutaloe.org/resources/Aloe\%20Scientific\%20 Primer.pdf (October 2017)

Jin Z. M., C. H. Wang, Z. P. Liu and W. J. Gong (2007) Physiological and ecological characters studies on Aloe vera under soil salinity and seawater irrigation. Process Biochemistry 42:710-714 https://doi.org/10.1016/j.procbio.2006.11.002

Lata C. and M. Prasad (2011) Role of DREBs in regulation of abiotic stress responses in plants. Journal of Experimental Botany 62:4731-4748, https://doi.org/10.1093/jxb/err210

Lee S. K. (2006) Overview of aloe study. In: New Perspectives on Aloe. Y. L. Park and S. K. Lee (eds.). Springer. New York, USA. pp:1-5.

Moghbeli E., S. Fathollahi, H. Salari, G. Ahmadi, F. Saliqehdar, A. Safari and M. S. H. Grouh (2012) Effects of salinity stress on growth and yield of Aloe vera L. Journal of Medicinal Plants Research 6:3272-3277, https://doi.org/10.5897/JMPR11.1698

Munns R. and M. Tester (2008) Mechanisms of salinity tolerance. Annual Review of Plant Biology 59:651-681, https://doi.org/10.1146/ annurev.arplant.59.032607.092911

Murillo-Amador B., A. Nieto-Garibay, E. Troyo-Diéguez, J. L. GarcíaHernández, L. Hernández-Montiel and R. D. Valdez-Cepeda (2015) Moderate salt stress on the physiological and morphometric traits of Aloe vera L. Botanical Sciences 93:639-648, https://doi org/10.17129/botsci.73

Nobel P. S. and H. Zhang (1997) Photosynthetic responses of three codominant species from the North-western Sonoran Desert - a $\mathrm{C}_{3}$ deciduous sub-shrub, a $\mathrm{C}_{4}$ deciduous bunchgrass, and a CAM evergreen leaf succulent. Australian Journal of Plant Physiology 24:787-796, https://doi.org/10.1071/PP96127

Núñez-Colima J. A., A. Pedroza-Sandoval, R. Trejo-Calzada, I. Sánchez-Cohen and R. Mata-González (2018) Effect of biofertilizers on growth of aloe (Aloe barbadensis Miller) and gel quality under different soil moisture contents. Revista Chapingo Serie Horticultura 24:27-40, https://doi.org/10.5154/r.rchsh.2017.02.013
Olfati J. A., E. Moqbeli, S. Fathollahi and A. Estaji (2012) Salinity stress effects changed during Aloe vera $\mathrm{L}$. vegetative growth. Journal of Stress Physiology and Biochemistry 8:152-158.

Pedroza S. A. y F. Gómez L. (2006) La Sábila (Aloe spp.). Propiedades, Manejo Agronómico, Proceso Agroindustrial y de Mercado. Universidad Autónoma Chapingo. Chapingo, Estado de México. $209 p$

Pedroza S. A., F. Gómez L., J. A. Samaniego-Gaxiola, R. Trejo C. y J. Ruiz T. (2009) Caracterización del proceso de industrialización y comercialización de la sábila: estudio de caso. Revista Chapingo Serie Zonas Áridas 8:241-246

Pedroza-Sandoval A., C. G. Aba-Guevara, J. A. Samaniego-Gaxiola, R. Trejo-Calzada, I. Sánchez-Cohen y J. A. Chávez-Rivero (2015) Características morfométricas y calidad de gel en sábila (Aloe barbadensis M.) aplicando algaenzimas y composta. Revista Mexicana de Ciencias Agrícolas 6:7-18.

Rahi T. S., K. Singh and B. Singh (2013) Screening of sodicity tolerance in Aloe vera: an industrial crop for utilization of sodic lands. Industrial Crops and Products 44:528-533, https://doi. org/10.1016/j.indcrop.2012.10.001

Ramachandra C. T. and P. S. Rao (2008) Processing of Aloe vera leaf gel: a review. American Journal of Agricultural and Biological Sciences 3:502-510, https://doi.org/10.3844/ajabssp.2008.502.510

Ritzema H. P. (2016) Drain for gain: managing salinity in irrigated landsA review. Agricultural Water Management 176:18-28, https:// doi.org/10.1016/j.agwat.2016.05.014

Roy S. J., S. Negrão and M. Tester (2014) Salt resistant crop plants. Current Opinion in Biotechnology 26:115-124, https://doi.org/10.1016/j. copbio.2013.12.004

Sánchez-Machado D. I., J. López-Cervantes, R. Sendón and A. SanchesSilva (2017) Aloe vera: ancient knowledge with new frontiers Trends in Food Science and Technology 61:94-102, https://doi. org/10.1016/j.tifs.2016.12.005

SAS Institute (2011) SAS/STAT® 9.3 User's Guide. SAS Institute Inc., Cary, North Carolina, USA. 8621 p.

SEMARNAT, Secretaria de Medio Ambiente y Recursos Naturales (2002) Norma Oficial Mexicana NOM-021-RECNAT-2000 Que establece las especificaciones de fertilidad, salinidad y clasificación de suelos. Estudios, muestreo y análisis. Diario Oficial de la Federación, 31 de diciembre de 2002. México, D. F.

SMN, Servicio Meteorológico Nacional (2017) Normales climatológicas por estado. 1951-2010. Servicio Meteorológico Nacional. Ciudad de México. https://smn.conagua.gob.mx/es/climatologia/ informacion-climatologica/normales-climatologicas-porestado (Septiembre 2017)

SSA, Secretaría de Salud (1995) Norma oficial mexicana NOM-117SSA1-1994, Bienes y servicios. Método de prueba para la determinación de cadmio, arsénico, plomo, estaño, cobre, fierro, zinc y mercurio en alimentos, agua potable y agua purificada por espectrometría de absorción atómica. Diario Oficial de la Federación. 29 de junio de 1995. México, D. F.

Wang Y. T. and K. J. Strong (1995) A two-year study monitoring several physical and chemical properties of field-grown Aloe barbadensis Miller leaves. Subtropical Plant Science 47:34-38.

Winter K., J. Aranda, and J. A. M. Holtum (2005) Carbon isotope composition and water-use efficiency in plants with crassulacean acid metabolism. Functional Plant Biology 32:381-388, https://doi. org/10.1071/FP04123 
